\title{
Study on the Effect of Herba Sarcandrae on Proliferation and Apoptosis of Prostate Cancer DU -145 Cell
}

\author{
Xiuxia Wang ${ }^{1, a}$ \\ a email \\ ${ }^{1}$ Luohe Medical College, Luohe, Henan, 462000
}

Keywords: Herba Sarcandrae; DU -145 Cells; Proliferation; Cell Cycle; Apoptosis

Abstract. By exploring statistical process control (SPC) technology in the printing and packaging.

\section{Introduction}

Prostate cancer (Prostate Cancer, $\mathrm{PCa}$ ) is a serious threat to the health of older men disease is the most common malignant tumors of the world's four most common cancer in men and older men. In the United States, PCa incidence of lung cancer has been exceeded, becoming a tumor harm men's health; and in our country, PCa male morbidity and mortality is the fastest-growing tumor. Patients with prostate cancer after 10 to 30 months, the median time to almost all hormone therapy is no longer sensitive to hormone therapy, converted to hormone-dependent cancers, hormone-independent prostate cancer clinical difficulties and focus. There are three kinds of human prostate cancer cells, LNCAP, PC -3 and DU - 145 cells, of which the latter two are non-hormone-dependent cells. In this study, traditional Chinese medicine treatment of cancer single herb fundamental treatment method, using the method of in vitro cell culture, explore Herba Sarcandrae on human prostate cancer DU - 145 Effect of cell proliferation and apoptosis.

\section{Materials and Methods}

Herba Sarcandrae experimental drug capsules by Ji Lin Junhong Pharmaceutical Co., Ltd., a brown solid powder capsules, size 0. 35g / tablets, batch number: 20090701. Control drug: LY294002, from the Tianjin Bin Xin Us Technology Development Co., Ltd., as a pale yellow solid powders, specifications 10mg / bottle, Item No: S110501.

Experimental drugs formulated 0. 35g / granulocyte Herba Sarcandrae a medium dissolved in $35 \mathrm{~mL} 1640$, with a diameter of $022 \mu \mathrm{m}$ filter sterilized sterile filter; and diluted to a final concentration of $0.1 \mathrm{mg} / \mathrm{mL}$, Pro preparation before use. 3mg L Y294002 10mL dissolved in DMSO (dimethyl sulfoxide) solution; diluted before use to a concentration $100 \mu \mathrm{mol} \mathrm{L} /$ prepared before use.

Grouping and processing sample group: the culture plate medium aspirating, divided into 7 groups was changed (ie blank group, negative control group, Sarcandrae solution 2. 5,1 25,0. 625mg / mL concentration group, LY29400225 $\mu \mathrm{mol} /$ L group and DMSO solvent control group) five wells, set $48 \mathrm{~h}$ to the observation point. Sample: (1) control group: $10 \%$ fetal calf serum RPMI 1640 complete medium $200 \mu \mathrm{L}$. (2) negative control group: DU - 145 cell suspension $160 \mu \mathrm{L}+$ saline $40 \mu \mathrm{L}$. (3) Herba Sarcandrae 2. $5 \mathrm{mg} / \mathrm{mL}$ group: DU - 145 cell suspension $160 \mu \mathrm{L}+2.5 \mathrm{mg} /$

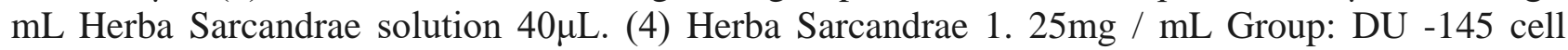
suspension $160 \mu \mathrm{L}+1.25 \mathrm{mg} / \mathrm{mL}$ Herba Sarcandrae solution 40 $\mu \mathrm{L}$. (5) Herba Sarcandrae 0. 625mg / mL group:. DU - 145 cell suspensions $160 \mu \mathrm{L}+0$ 625mg / mL Herba Sarcandrae solution 40 $\mu \mathrm{L}$. (6) LY294002 group: DU - 145 cell suspension $160 \mu \mathrm{L}+25 \mu \mathrm{mol} / 1 \mathrm{LY} 29400240 \mu \mathrm{L}$. (7) DMSO solvent control group: DU - 145 cell suspension $160 \mu \mathrm{L}+\mathrm{DMSO}$ solvent $40 \mu \mathrm{L}$ (due to the control drug LY294002 insoluble in water, it is dissolved in DMSO, provided that the solvent DMSO control group is used to exclude itself on the cells).

DU -145 human prostate cancer cells (West China Medical School) provided by the Science and Technology Center, West China Hospital of Sichuan University, built by the American ATCC strains, the cell line has no detectable androgen sensitivity, androgen-independent cells. Containing 10\% 
fetal bovine serum RMPI -1640 broth placed in $37{ }^{\circ} \mathrm{C}$, the incubator with $5 \%$ CO2 culture, every 2 to 3 days medium was changed once a week passaged 2 to 3 times, passage ratio 1: 3 .

Group 7 with formulated liquid acts on DU -145 cells $48 \mathrm{~h}$ later, after washing with PBS, fixed with methanol, the next 40 optical microscope.

Withdrawing the cell culture medium was discarded after the drug, rinsed with PBS three times, for culture maintenance medium with new cells. $50 \mu \mathrm{L}$ MTT solution was added to each well $(5 \mathrm{mg} /$ mL, i.e. 0. 5\% MTT), culture was continued 48h. Pei termination

After the raise, squirt carefully aspirate the culture medium. Each hole by adding $150 \mu \mathrm{L}$ DMSO, set shaker speed oscillation $10 \mathrm{~min}$, the crystal was fully dissolved. Each well was measured in a microplate reader OD570nm absorbance (OD) value.

After the cells were cultured 48h, removed from the incubator, with D - Hank's balanced salt solution wash 2 or 3 times to prepare cell suspension using a hemocytometer count, the cell count reached $1 \times 106 / \mathrm{mL}$. Then cells were washed with PBS buffer was added 2 to 3 times, 1000r / min, centrifuged $5 \mathrm{~min}$, the supernatant discarded, and then fixed with $75 \%$ ethanol was added dropwise, and then 1\% TritonX100 processing 10min, RnaselmL for 10min propidium bromide (PI ) staining 30min, used by the US company EliteESP type Coulter flow cytometry analysis to calculate the proportion of apoptosis and cell cycle distribution.

All information using SPSS 17. 0 for statistical analysis, the results were presented as mean \pm standard deviation $(\mathrm{x} \pm \mathrm{s}$ ) represented. Among the groups were compared using ANOVA, where homogeneity of variance were used LSD method were missing variance with Dunnett's test.

\section{Results}

Human prostate cancer DU -145 cells after $48 \mathrm{~h}$ in each group after the chemical treatment, reduce the number of performance, volume is reduced, the cells to varying degrees rough edges, chromosome condensation or karyopycnosis fragmentation, accumulation of dead cells gathered to Sarcandrae high solution the most significant concentrations.

To each well of the absorbance (OD) on behalf of the proliferative activity of cells. OD higher the value, the more the number of viable cells, indicating that the stronger the proliferation inhibition worse. Data shows, the negative control group OD value significantly higher than the control group $(\mathrm{P}<0.01)$, indicates that DU - 145 cells actively proliferating; Herba Sarcandrae solution 2. 5,1 $25 \mathrm{mg} / \mathrm{mL}$ and LY294002 $25 \mu \mathrm{mol} / \mathrm{mL}$ concentrations OD. were lower than the negative control group, and showed a highly significant difference $(\mathrm{P}<0.01)$, Sarcandrae solution 0 . $625 \mathrm{mg} / \mathrm{mL}$ concentration group OD values are different than the negative control group $(\mathrm{P}<0.05)$. Herba Sarcandrae solution 2. 5mg / mL high dose group of DU -. Inhibitory potency of 145 cells was significantly higher than Herba Sarcandrae 25,0 625mg / mL, the low concentration group and LY29400225 $\mu \mathrm{mol} / \mathrm{mL}$ concentration group $(\mathrm{P}<0.01)$, while Sarcandra, the low-dose group and LY294002 $25 \mu \mathrm{mol} / \mathrm{mL}$ concentrations to inhibit the effectiveness of DU -145 cells basically the same, no difference. The OD value of the DMSO solution in the control group was significantly lower than the negative control group $(\mathrm{P}<0.01)$.

The results showed that Herba Sarcandrae solution with high, medium and low three concentrations inhibited cell proliferation DU -145, for their ability to inhibit cell dose-dependent, high concentrations of DU -145 suppressor cells was stronger than low concentration group and LY294002 $25 \mu \mathrm{mol} / \mathrm{mL}$ concentrations. The OD value of the DMSO solvent control group was lower than the negative control group reasons may be DMSO cell itself has some toxicity, but it does not completely hide LY294002 inhibition of cell proliferation of DU -145.

Flow cytometry (FCM) detection Herba Sarcandrae solution and LY294002 on DU - 145 cell cycle distribution. Flow cytometry (FCM) detection Delisheng influence and etoposide on DU -145 cell cycle distribution.

Data show that the concentration of each solution Sarcandrae group and LY294002 group of DU -145 cells G0 G1 phase had no effect ( $>>0$ 0 05); Herba Sarcandrae solution of each concentration group and LY294002 group can make DU -145 cells S period decreased (P <0. 01,0. 05), G2 M 
phase increased $(\mathrm{P}<0.01,0.05)$.

According to the above results can be speculated Sarcandrae solution of each concentration group and LY294002 group of DU - 145 cells in G2 M phase arrest and five reduced role, so that the cell can not enter the next cycle, and inhibits cell proliferation. The DMSO Although there is some inhibition of cell proliferation, but had no effect on the cell cycle, Guck that the impact of LY294002 group dissolved in DMSO on cell cycle itself entirely from LY294002.

Flow cytometry (FCM) detection Herba Sarcandrae solution and LY294002 on DU - 145 cell apoptosis, and calculate the rate of apoptosis (\%). Herba Sarcandrae solution on DU -145 apoptosis rates.

Experimental data shows: Herba Sarcandrae solution of each concentration group and LY294002 group apoptosis rate was significantly higher than the negative control group $(\mathrm{P}<0.05,0$ 01.). The results showed that the concentration of each solution Sarcandrae group and LY294002 group can induce apoptosis in DU -145. Herba Sarcandrae solution induced apoptosis DU -145 cells in a certain dose-effect relationship. The DMSO Although there is some inhibition of cell proliferation, but had no effect on apoptosis, Guck that the impact of LY294002 group dissolved in DMSO apoptosis itself entirely from LY294002.

\section{Discussion}

Prostate cancer (PCa) the occurrence and development is closely related to androgen stimulation, $80 \%$ of newly diagnosed prostate cancer patients on androgen deprivation therapy sensitive, but more than $50 \%$ of patients will relapse, and converted to hormone-independent. The exact mechanism by the androgen-dependent prostate cancer cells into a non-dependent growth is not yet fully understood. Currently endocrine therapy and radical prostatectomy treatment of early androgen dependent prostate cancer is effective. However, androgen-independent prostate cancer treatment more difficult. So choose the subject of androgen-independent prostate cancer study. Including prostate cancer cell lines LNCaP, PC - 3 and DU - 145 three. LNCaP androgen-dependent cancer cells, DU - 145 and PC - 3 cells to androgen-dependent cancer cell lines. The Institute selected DU -145 cell line that does not have a detectable androgen-sensitive,

Of androgen insensitivity. Chinese medicine nonaggressive prostate cancer can be summarized as "Dysuria", "disease product." According to "Classic" and "kidney aging, said," Zhang Jingyue's "kidney gasification negative," said Zhang Zhongjing, Jingyue, Yu Tuan of "blood stasis channel negative," said Wang Ren of "blood stasis caused Archives of," said Zhu Dan's " phlegm tumorigenic, "said Ko Kam tribunal" are virtual phlegm carcinogenic, "said Hua Tuo" toxin-tumorigenic, "said combining the characteristics of prostate cancer incidence, clinical manifestations and pathological changes locally, we believe that the disease is essential pathogenesis of righteousness deficiency, Tanning drug accumulation, Zhengjia accumulation. When Qi righting therapy, blood disease elimination, detoxification. The experimental observation of a single herb Herba Sarcandrae on human prostate cancer DU - 145 Effect of cell proliferation and apoptosis. Herba Sarcandrae the "People's Republic of China Pharmacopoeia" collection contains the drugs, with heat and cooling blood, Qufeng network, blood Xiaoban role; modern medicine pharmacological studies found to have anti-tumor, anti-bacterial (virus) anti-inflammatory and enhance immunity role, in line with the basic pathogenesis of prostate cancer, for its treatment. Through a large number of experimental and clinical studies have confirmed that Herba Sarcandrae of murine and human tumors inhibit multiple systems, especially Huang Zhifeng and other prostate cancer patients transurethral resection, while testicular resection, oral endocrine drugs Herba Sarcandrae intramuscular injection, based on the results found that significantly reduces PSA levels after 15 months, the impact of FPSA (free prostate specific antigen) / PSA percentage, and improved clinical symptoms of prostate symptoms. Description Herba Sarcandrae whether from the pharmacological effects or clinical efficacy of all tumors, including prostate cancer have a good inhibition. But the experimental study Herba Sarcandrae effect on human prostate cancer cells has not yet been carried out, so this experiment is Herba Sarcandrae whole experimental treatment for prostate cancer medicine useful supplement, while the pre-clinical studies have also undertaken to 
provide the present experiment theoretical support. LY294002 is a chemical formulation, a molecular weight of 307. 3, the formula C19H17NO3. The study showed that LY294002 can inhibit the growth and proliferation of lung cancer cells and gastric cancer AGS cells in a time- and dose-dependent mechanism may be cell cycle arrest and induction of apoptosis. Thus, this experiment as a positive control to comparative illustration Herba Sarcandrae DU -145 prostate cancer cell proliferation, cell cycle and apoptosis.

The results of this experiment showed that: Herba Sarcandrae solution enables human prostate cancer DU - 145 cells showed morphological changes of apoptosis, inhibition of cell proliferation, inhibition of cell cycle progression and induce apoptosis, and showed dose-effect relationship, but for DU - mechanism of action above 145 cells for further study.

\section{Acknowledgements}

This is the 2015 Henan Province Science and Technology Department of Henan Province basis frontier planning project milestones. Item Number: 152300410015.

\section{References}

[1] Liu Youfang, Zhang Yaqiang. Integrative Urology [M]. Beijing: People's Medical Publishing House, 2007: 385.

[2] Zheng Ying, Wu Chunxiao, Jin Fan, et al. Shanghai area 1973-2005 annual incidence trends in cancer [J]. Diagnosis Theory and Practice, 2009,8 (1): 25-32.

[3] Li Anle, Shang Hanji, Li Rongmin, et al. Yangpu District 1980-2005 annual cancer deaths Trend Analysis [J]. Chinese tumors, 2009,18 (10): 818-822.

[4] Eder I E, Culig Z, Ramoner R, et al. Inhibition of LNCaP prostatecancer cell by means of androgen receptor antisense oligonucleotides

[J]. Cancer Gene Ther, 2000,7: 997.

[5] Isaacs J T. The biology of hormone refractory prostate cancer. Whydoes it develop [J]. Urol Clin North Am, 1999,26: 263 - 273.

[6] Huang Zhifeng Lai Haibiao, Zeng Ye.. Herba Sarcandrae injection on 26 cases of advanced prostate cancer, PSA F, - PSA influence [J]. Traditional Chinese Medicinal Research, 2006,19 (11): 25 - 27.

[7] Xie Zihui, Sun Xiuhua, Ke Dan. LY294002 of cell proliferation in lung cancer cell lines H460 and LK2 influence and SKP2 and p27 expression in [J]. Chinese Journal of Cancer Prevention and Control, 2010,17 (3): 182-- 185,205.

[8] Tang Mei, Chen Weichang, Li Rui. LY294002 for gastric cancer cell proliferation and invasion of human [J]. Suzhou University: Medicine Edition, 2010,30 (2): 347-350. 